\title{
Therapeutic anti-glioma effect of the combined action of PCSK inhibitor with the anti-tumoral factors secreted by Poly (I:C)- stimulated macrophages
}

\author{
Mélanie Rose $\mathbb{D}^{1,2} \cdot$ Marie Duhamel $\mathbb{D}^{1} \cdot$ Soulaimane Aboulouard $\mathbb{D}^{1} \cdot$ Firas Kobeissy $\mathbb{D}^{3} \cdot$ Dominique Tierny $^{2} \cdot$ \\ Isabelle Fournier $\mathbb{D}^{1,4} \cdot$ Franck Rodet $\mathbb{D}^{1} \cdot$ Michel Salzet $\mathbb{C}^{1,4}$
}

Received: 9 September 2020 / Revised: 27 November 2020 / Accepted: 8 December 2020 / Published online: 5 January 2021

(c) The Author(s), under exclusive licence to Springer Nature America, Inc. part of Springer Nature 2021. This article is published with open access

\begin{abstract}
Macrophages plasticity is a key feature in cancer progression. Neoplastic cells can alter their immune functions and orient them into a pro-tumoral phenotype. In this context, we developed a new therapeutic strategy to switch macrophages phenotype and reactivate their anti-tumoral functions. We showed a dual activity of a proprotein convertases inhibitor as anti-glioma drug and anti-tumoral macrophages' reactivation drug. Proprotein convertases are proteases that cleave proteins into functional proteins. Several of their substrates are involved in tumorigenesis and immunosuppression. We combine here proprotein convertases inhibitor with Poly (I:C), a TLR3 ligand, to increase the anti-tumoral activity of macrophages. With mass spectrometry-based proteomics, system biology, combined with biological assays, we established that a stimulation of macrophages with Poly (I:C) increased their secretion of pro-inflammatory cytokines and anti-tumoral factors. 3D invasion assay showed the efficacy of these anti-tumoral factors against mixed glioma cells and macrophages spheroids. Besides, immunofluorescence and proliferation assays showed an additive effect of the proprotein convertases inhibitor and the antitumoral factors secreted by Poly (I:C)-treated macrophages on both anti-glioma activity and macrophages anti-tumoral orientation directly in tumor microenvironment, leading to an innovative glioma therapy.
\end{abstract}

\section{Introduction}

Oncoimmunology is a growing field. Tumors present a complex microenvironment in which several cell types can be found. Among them, immune cells such as macrophages,

These authors contributed equally: Mélanie Rose, Marie Duhamel

Supplementary information The online version of this article (https:// doi.org/10.1038/s41417-020-00286-1) contains supplementary material, which is available to authorized users.

Michel Salzet

michel.salzet@univ-lille.fr

1 Université Lille, INSERM, U1192 - Laboratoire Protéomique, Réponse Inflammatoire et Spectrométrie de Masse-PRISM, F59000 Lille, France

2 Oncovet Clinical Research (OCR), SIRIC ONCOLille, Villeneuve d'Ascq, France

3 Department of Psychiatry, McKnight Brain Institute, University of Florida, Gainesville, FL 32611, USA

4 Institut Universitaire de France, 75000 Paris, France microglia, T, B, and NK lymphocytes are present [1]. Most of the current strategies target $\mathrm{T}$ lymphocytes to reactivate their cytotoxic response. In cancer, T lymphocytes response is inhibited via the recognition of the PD1 receptor located on T lymphocytes and its ligand PDL1 expressed by cancer cells and pro-tumor macrophages [2]. Yet the full activation of these lymphocytes requires the reactivation of pro-tumor macrophages to express a pro-inflammatory and anti-tumor phenotype. Thus, it is necessary to act before this PD1/ PDL1 pathway. Cancer cells secrete different antiinflammatory factors that create an immunosuppressive environment and participate in the recruitment of macrophages. These macrophages are diverted from their antitumor function. They participate in tumorigenesis by improving metastatic and angiogenic processes and by inhibiting cytotoxic response [3]. Macrophages must keep their pro-inflammatory phenotype to re-exert their phagocytic activity and to activate the anti-tumor immune response. In this context, proprotein convertases seem to be a clear target. Proprotein convertases (PCs) are proteases of the subtilisin-kexin family that cleave proproteins through limited proteolysis and convert them into bioactive proteins 
and peptides [4-6]. Mammalian PCs include PCSK1, PCSK2, furin, PCSK4, PCSK5, PCSK6, and PCSK7 which are known to cleave proproteins at paired basic residues [7]. PCs cleave a variety of precursor proteins within the secretory pathway, including neuropeptides, hormones, growth factors, and their respective receptors, adhesion molecule, bacterial toxins, and viral glycoproteins [8]. However, deregulation of these enzymes has been associated with pathological conditions including endocrinopathies [9], Alzheimer's disease [10], and tumors [11]. Proprotein convertases are implicated in malignancies. PCSKs promote cancer growth and EMT transition [12, 13] by activating tissue-modifying enzymes through at least matrix metalloproteinases or by inhibiting growth factors regulators [4]. The PCSK upregulation is correlated with accelerated tumor progression and poor prognosis. Furin and PACE4 are the most implicated in ovarian [14], prostate cancers [15] and glioma [16]. In this context, inhibiting PCSK/Furin activity has emerged as a therapeutic approach for suppressing cancerous cell-growth and metastatic activity [17, 18]. The specific Furin inhibition using a bifunctional GM-CSF-Furin shRNA construct and an in vitro transduction protocol on the patient's cancer cells have been efficacious and well-tolerated in phase I-II trials with advanced Ewing's sarcoma [19] and ovarian cancer patients [20]. Several PCs inhibitors exist such as $\alpha 1$-PDX or inhibitors of PACE4 which reduced tumor progression $[18,21]$. In our previous study, we have demonstrated that a peptidomimetic PC inhibitor, that can act against furin and on other PCs such as PC1/3, can reactive macrophages without toxicity and reduce glioma growth [22] and has the advantage to have a broad spectrum on PCs.

However, another way to get anti-tumoral activity has recently emerged, which corresponds to the immune response reactivation.

In this context, it has been demonstrated that Furin and the proprotein convertase $1 / 3(\mathrm{PC} 1 / 3)$ are two complementary targets for reinforcing the immune response [23-30]. Furin is critical for the maintenance of peripheral $\mathrm{CD} 4+$ Foxp $3+\mathrm{T}$ regulatory cell-dependent immune tolerance and normal $\mathrm{CD} 4+\mathrm{T}$ helper $(\mathrm{Th})$ cell polarization in vivo $[30,31]$. We demonstrated that $\mathrm{PC} 1 / 3$ knock-out mice have high plasma levels of pro-inflammatory cytokines [25]. We also showed that a rat macrophage cell line (NR8383) PC1/3 knockdown (KD) secretes a high concentration of pro-inflammatory chemokines and cytokines [27]. These secreted factors have chemotactic and antitumor properties. When stimulated with TLRs ligands, PC1/ $3 \mathrm{KD}$ macrophages are over-activated and their immune response is exacerbated [26, 27]. Secreted factors by these PC1/3 KD macrophages after TLR4 stimulation with LPS and the anti-tumoral agent Paclitaxel have anti-tumor activities against breast, ovarian, and glioma cancer cells lines. $\mathrm{PC} 1 / 3 \mathrm{KD}$ macrophages can inhibit invasion of glioma cancer cells (C6 and F98) in 3D spheroid mixed macrophages-glioma culture [26]. We established that PC1/ $3 \mathrm{KD}$ macrophages secrete high quantities of extracellular vesicles (EVs) [25, 27, 29] which is even more important when TLR4 or TLR9 pathways are triggered in PC1/3 KD macrophages [27, 28]. PC1/3 modulates GRAMD4 levels and thus regulate TLR9 trafficking, to modulate the inflammatory response [29]. Its inhibition reactivates the pro-inflammatory TLR MYD88 NFKB dependent pathway while the anti-inflammatory STAT3 pathway is downregulated [29].

Taken together, these results point out that $\mathrm{PC} 1 / 3 \mathrm{KD}$ macrophages can secrete factors that can exert anti-tumoral activity. Thus, we developed a strategy based on PCSK inhibitor to both inactivate the tumor cells and reactivate the tumor associated macrophages (TAM). We established this double effect and the clear ability to reactivate TAM [22]. However, in glioma, the antiviral pathway seems to be more implicated than the TLR4 pathway [32]. We thus investigated in the present paper, the ability to reactivate the anti-tumoral response of macrophages by combining PCSK inhibitor with Poly (I:C) stimulation as a potential anti-glioma therapy. We have demonstrated the secretion by macrophages of antitumoral factors. We have confirmed the combined effect of the treatment by inhibiting tumor growth and reactivating macrophages in mixed spheroids. A graphical abstract describing the proteomic analysis we performed throughout this study is presented in Fig. 1.

\section{Materials and methods}

\section{Experimental design and statistical rationale}

Shotgun proteomics experiments were conducted in biological triplicates $(n=3)$. Spheroid studies were conducted in biological triplicates $(n=3)$ as well as all biological assays. Preactivation spheroid studies and Poly (I:C) treatedNCH82 spheroids were conducted in biological duplicates $(n=2)$ as well as shotgun proteomics experiments on conditioned media-treated NR8383. For the proteomics statistical analysis, extracted proteins or secreted medium proteins presenting as significant by the ANOVA test analysis were used ( $p$-value $<0.05)$. Normalization was achieved using a Z-score with matrix access by rows. For invasion tests, results obtained were depicted through a box plot figure. Significant differences were identified using Tukey's multiple comparisons test where: $* P \leq 0.05 ; * * P \leq$ $0.01 ; * * * P \leq 0.001 ; * * * * P \leq 0.0001$. The tests were made based on an equal variance between the groups. Error bars are represented as s.d. 
Fig. 1 Experimental strategy for proteomic analysis. Graphic summary of the proteomics experiments conducted in this study and the main results.

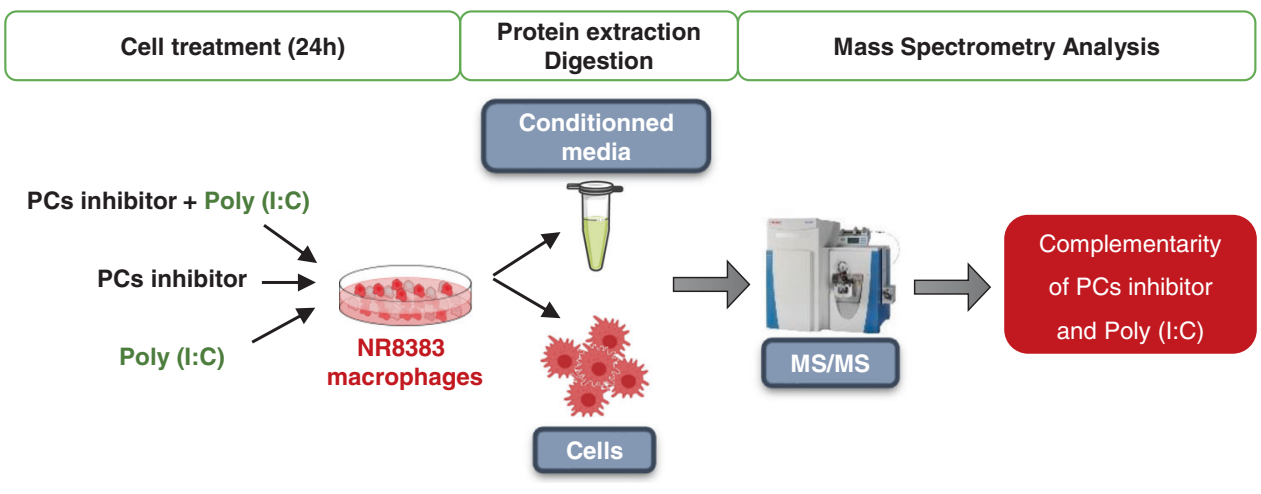

\section{Chemicals and reagents}

Water $\left(\mathrm{H}_{2} \mathrm{O}\right)$, formic acid $(\mathrm{FA})$, acetonitrile $(\mathrm{ACN})$, and trifluoroacetic acid (TFA) were obtained from Biosolve B. V. (Dieuze, France). 2,5 dihydroxybenzoic acid (DHB), ProteoMass MALDI Calibration kit, DL-dithiothreitol (DTT), thiourea, Temozolomide (TMZ), PCs inhibitor (ref. 537076) and iodoacetamide (IAA) were purchased from Sigma-Aldrich (Saint-Quentin Fallavier, France). LysC/ Trypsin was purchased from Promega (Charbonnieres, France). Polylysine-coated slides, Dulbecco's Modified Eagle's Medium (DMEM), Ham's F12K, heat-inactivated fetal bovine serum (FBS), trypsin, and phosphate buffer saline (PBS), penicillin and streptomycin were purchased from Thermo-Scientific (Braunschweig, Germany). Poly (I: C) was purchased from Invivogen (San Diego, California, USA). NR8383 is a rat alveolar macrophage cell line (CRL2192) obtained from ATCC (Manassas, Virginia, USA). The rat C6 glioma cell line was kindly provided by Prof. Dr. Bernd Kaina (Institute of Toxicology, University Medical Center, Mainz, Germany).

\section{Cell culture}

Rat alveolar NR8383 macrophages were cultured in Ham's F12K medium supplemented with $15 \%$ fetal bovine serum and $1 \%$ penicillin/streptomycin (100 units per ml). Rat glioma C6 cells and human glioma cell line $\mathrm{NCH} 82$ were cultured in DMEM and supplemented with $10 \%$ FBS, $1 \%$ Lglutamine $(2 \mathrm{mM})$, and $1 \%$ penicillin/streptomycin (100 units per ml). All cell lines were cultured at $37^{\circ} \mathrm{C}$ in a humidified atmosphere $\left(5 \% \mathrm{CO}_{2}\right)$.

\section{Cell supernatant collection and total protein extraction}

NR8383 cells were plated on sterile 24 well plates and cultured until they reached confluence. For stimulation, cells were starved overnight in Ham's F12K medium supplemented with $2 \%$ FBS. Cells were then stimulated in serum-free medium with different concentrations of Poly (I: C) $(5,10$, or $15 \mu \mathrm{g} / \mathrm{ml})$. Also, cells were stimulated with $100 \mu \mathrm{M}$ of PCs inhibitor-associated or not with $10 \mu \mathrm{g} / \mathrm{ml}$ of Poly (I:C) in serum-free medium. At $24 \mathrm{~h}$, cell supernatants were centrifuged at $500 \times g$ and passed through a $0.22-\mu \mathrm{m}$ filter to remove cells and debris. Four hundred microliters of the cell supernatant were collected for each condition. Cells were washed three times with ice-cold PBS and then lysed with RIPA buffer for total protein extraction $(150 \mathrm{mM}$ $\mathrm{NaCl}, 50 \mathrm{mM}$ Tris, $5 \mathrm{mM}$ EGTA, $2 \mathrm{mM}$ EDTA, $100 \mathrm{mM}$ $\mathrm{NaF}, 10 \mathrm{mM}$ sodium pyrophosphate, $1 \%$ NP40, $1 \mathrm{mM}$ PMSF, and $1 \mathrm{X}$ protease inhibitors). After $3 \times 30 \mathrm{~s}$ sonication, cell debris was removed by centrifugation $(16,000 \times g$, $10 \mathrm{~min}, 4^{\circ} \mathrm{C}$ ), supernatants were collected and protein concentrations were measured using a Bio-Rad Protein Assay Kit, according to the manufacturer's instructions.

\section{Filter-aided sample preparation (FASP)}

The samples were processed using a shotgun bottom-up proteomic approach. Total protein extract $(0.04 \mathrm{mg})$ was used for FASP analysis as described previously [33-35]. We performed FASP using Microcon devices YM-10 from Millipore (Burlington, Massachusetts, USA) before adding Lys-C trypsin for protein digestion $(40 \mu \mathrm{g} / \mathrm{mL}$ in $0.05 \mathrm{M}$ Tris- $\mathrm{HCl})$. An equivalent volume of reduction solution (DTT $0.1 \mathrm{M}$ ) was added to each sample followed by an incubation step at $95{ }^{\circ} \mathrm{C}$ for $15 \mathrm{~min}$. Then the samples were processed following the filter-aided sample preparation (FASP) protocol using a filter with a nominal molecular weight limit of $10,000 \mathrm{Da}$ (Amicon Ultra-0.5 $10 \mathrm{~K}$, Millipore). Briefly, each sample was mixed with $200 \mu \mathrm{L}$ of denaturant buffer (8 M urea, Tris/ $\mathrm{HCl} 0.1 \mathrm{M}, \mathrm{pH} 8.5)$ and transferred to FASP filters. The samples were centrifuged at $14,000 \times \mathrm{g}, 20^{\circ} \mathrm{C}$, for $15 \mathrm{~min}$. For the alkylation step, $100 \mu \mathrm{L}$ of $0.05 \mathrm{M}$ of iodoacetamide in denaturant buffer was added to each sample, followed by incubation in the dark for $20 \mathrm{~min}$ at room temperature. Samples were washed twice with $100 \mu \mathrm{L}$ of denaturant buffer followed by two washes with $100 \mu \mathrm{L}$ of buffer $\mathrm{AB}$ (Ammonium bicarbonate 
$0.05 \mathrm{M})$. After each washing step, centrifugation was performed at $14,000 \times \mathrm{g}, 20^{\circ} \mathrm{C}$, for $15 \mathrm{~min}$. The proteins were digested by adding $40 \mu \mathrm{L}$ of trypsin at $40 \mu \mathrm{g} / \mathrm{ml}$ in buffer $\mathrm{AB}$, and then incubated at $37^{\circ} \mathrm{C}$ overnight. The peptides were eluted by adding $50 \mu \mathrm{L}$ of saline solution $(\mathrm{NaCl} 0.5 \mathrm{M})$ and centrifuged at $14,000 \times \mathrm{g}, 20^{\circ} \mathrm{C}$, for $15 \mathrm{~min}$. The digestion was stopped by adding $10 \mu \mathrm{L}$ of TFA $5 \%$. The samples were desalted using ZipTip C-18 (Millipore) and eluted with a solution of ACN/0.1\% TFA (7:3, v/v). The samples were dried with SpeedVac and resuspended in $20 \mu \mathrm{L}$ of $\mathrm{ACN} / 0.1 \%$ formic acid $(0.2: 9.8, \mathrm{v} / \mathrm{v})$ just before processing using LC-MS/MS. Experiments were done in biological triplicate $(n=3)$.

\section{Proteomics analysis of the cell supernatants}

Supernatants volume obtained from NR8383 macrophages treated or not with PCs inhibitor or/and Poly (I:C) during $24 \mathrm{~h}$ were reduced to $100 \mu \mathrm{L}$ in a SpeedVac. Cell supernatants were denatured with $2 \mathrm{M}$ urea in $10 \mathrm{mM}$ HEPES, pH 8.0 by sonication on ice. The proteins were reduced with $10 \mathrm{mM}$ DTT for $40 \mathrm{~min}$ at $56^{\circ} \mathrm{C}$ followed by alkylation with $55 \mathrm{mM}$ iodoacetamide for $40 \mathrm{~min}$ in the dark. The iodoacetamide was quenched with $100 \mathrm{mM}$ thiourea. The proteins were digested with $1 \mu \mathrm{g} \mathrm{LysC/}$ Trypsin mixture (Promega) overnight at $37^{\circ} \mathrm{C}$. The digestion was stopped with $0.5 \%$ TFA. The samples were desalted using ZipTip C-18 (Millipore) and eluted with a solution of ACN/0.1\% TFA (7:3, v/v). The samples were dried with SpeedVac and resuspended in $20 \mu \mathrm{L}$ of ACN/ $0.1 \%$ formic acid $(0.2: 9.8, \mathrm{v} / \mathrm{v})$ just before processing using LC-MS/MS. Experiments were done in biological triplicate $(n=3)$.

\section{LC-MS/MS analysis}

Mass spectrometry proteomics analysis of digested proteins was performed using a nano Acquity UPLC system (Waters) coupled with the Q-Exactive Orbitrap mass spectrometer (Thermo Scientific) via a nanoelectrospray source. The samples were separated using online reversed-phase, using a preconcentration column (nanoAcquity Symmetry $\mathrm{C} 18,5 \mu \mathrm{m}, 180 \mu \mathrm{m} \times 20 \mathrm{~mm}$ ) and an analytical column (nanoAcquity BEH C18, $1.7 \mu \mathrm{m}, 75 \mu \mathrm{m} \times 250 \mathrm{~mm}$ ). The peptides were separated by applying a linear gradient of acetonitrile in $0.1 \%$ formic acid (5-35\%) for $2 \mathrm{~h}$, at a flow rate of $300 \mathrm{~nL} / \mathrm{min}$. The Q- Exactive was operated in datadependent mode defined to analyze the ten most intense ions of MS analysis (Top 10). The MS analysis was performed with an $\mathrm{m} / \mathrm{z}$ mass range between 300 to 1600 , resolution of 70,000 FWHM, AGC of $3 \mathrm{e} 6$ ions and maximum injection time of $120 \mathrm{~ms}$. The MS/MS analysis was performed with an $\mathrm{m} / \mathrm{z}$ mass range between 200 to 2,000;
AGC of $5 \mathrm{e} 4$ ion; maximum injection time of $60 \mathrm{~ms}$ and resolution set at 17,500 FWHM.

\section{Protein ID and Data analysis}

Proteins were identified by comparing all MS/MS data with the proteome database of the complete reviewed proteome of Rattus norvegicus (Uniprot, release July 2018; 8,054 entries), using the MaxQuant software version 1.6.1.0 $[36,37]$. Lys-C trypsin specificity was used for the digestion mode with two missed cleavages. Carbarmidomethylation of cysteines was set as a fixed modification. Nterminal acetylation and methionine oxidation were selected as the variable modifications. For MS spectra, an initial mass tolerance of $6 \mathrm{ppm}$ was selected, and the MS/MS tolerance was set to $20 \mathrm{ppm}$ for HCD data [38]. For identification, the FDR at the peptide spectrum matches (PSMs) and protein level was set to 0.01 . Relative, label-free quantification of proteins was performed using the MaxLFQ algorithm integrated into MaxQuant with the default parameters [39]. Analysis of the proteins identified was performed using Perseus software (http://www.perseus-framew ork.org/) (version 1.6.2.1) [40]. The file containing the information from identification was used with hits to the reverse database, and proteins identified with modified peptides and potential contaminants were removed. Then, the LFQ intensity was logarithmized $(\log 2[x])$. Categorical annotation of rows was used to define different groups depending on the concentration of PCs inhibitor used $(0,50$, $100,150 \mu \mathrm{M})$. Multiple-samples tests were performed using an ANOVA test with a $p$-value of 0.05 and preserved grouping in randomization. The results were normalized by Z-score and represented as hierarchical clustering. Functional annotation and characterization of identified proteins were obtained using PANTHER software (version 14.0, http://www.pantherdb.org) and STRING (version 10.5, http://string-db.org).

\section{Sub-network enrichment pathway analysis}

Using Elsevier's Pathway Studio (version 11.0/ /Elsevier), all relationships between the differentially expressed proteins among all conditions were depicted based on the Ariadne ResNet [41]. For proteins identified in the shotgun analysis post-stimulation of cell lines with PCs inhibitor, the Subnetwork Enrichment Analysis (SNEA) algorithm was used to detect the statistically significant altered biological pathways in which the identified proteins are involved. This algorithm uses Fisher's statistical test to detect any nonrandom associations between two categorical variables organized by a specific relationship. Also, this algorithm starts by creating a central "seed" from all the relevant identities in the database and builds connections with 
associated entities based on their relationship with the seed. SNEA compares the sub-network distribution to the background distribution using one-sided Mann-Whitney U-Test and calculates a $p$-value; thus, representing a statistical significance between different distributions. In all analyses that we performed, the GenBank ID was used to form experimental groups based on the different conditions present for analysis. The pathway networks were reconstructed based on biological processes and molecular functions for every single protein, along with its associated targets.

\section{Biological assays}

\section{Generation of complete and fractions NR8383 supernatants}

NR8383 macrophages were treated with or without $10 \mu \mathrm{g} /$ $\mathrm{mL}$ of Poly (I:C) in complete Ham's F12K for $24 \mathrm{~h}$. At $24 \mathrm{~h}$, cell supernatants were centrifuged at $200 \times \mathrm{g}$ and passed through a $0.22 \mu \mathrm{m}$ filter to remove cells and debris. Supernatants were then used for spheroids stimulation.

Supernatants were also fractionated with Amicon ${ }^{\circledR}$ Ultra Centrifugal Filters. The different molecular weight cut off were used $(100,50,30,10$ and $3 \mathrm{kDa})$ according to the manufacturer's instruction. The different fractions were used to stimulate C6 cells.

\section{Spheroid generation and embedding in a collagen matrix}

C6 rat glioma cells associate or not with NR8383 pretreated or not with $10 \mu \mathrm{g} / \mathrm{mL}$ of Poly (I:C) for $24 \mathrm{~h}$ were resuspended in complete Ham's F12K medium supplemented with $5 \%$ of collagen mixture at the final concentration of 8,000 cells of each cell line in $20 \mu \mathrm{L}$. For human glioma spheroids generation, we used 8000 NCH82 glioma cells. The collagen mixture was prepared by mixing $2 \mathrm{ml}$ of PureCol ${ }^{\circledast}$ bovine collagen type I solution $(3 \mathrm{mg} / \mathrm{mL}$; Advanced BioMatrix) with $250 \mu \mathrm{L}$ of $10 \mathrm{X}$ minimal essential medium (MEM) (Sigma-Aldrich) and $500 \mu \mathrm{L}$ of sodium hydroxide $0.1 \mathrm{M}$. Cells were cultivated in hanging-drop on the lid of a petri dish with PBS during $72 \mathrm{~h}$ at $37^{\circ} \mathrm{C}$ in a humidified atmosphere $\left(5 \% \mathrm{CO}_{2}\right)$. The newly formed C6 spheroids and mixed C6/NR8383 spheroids were then implanted in the center of each well of a 24-well plate coated with a collagen mixture described before (one spheroid per well in $400 \mu \mathrm{L}$ of collagen mixture per well). After cell spheroid embedding, the plate was incubated for $30 \mathrm{~min}$ at standard culture conditions to solidify the collagen. Thereafter $400 \mu \mathrm{L}$ of complete Ham's F12K medium or conditioned medium from NR8383 was overlaid on the collagen matrix in each well and supplemented with $100 \mu \mathrm{M}$ of PCs inhibitor. The complete system was incubated for a total of 4 to 6 days. Experiments were done in biological triplicate $(n=3)$. Human spheroids were treated with either $10 \mu \mathrm{g} / \mathrm{mL}$ Poly (I:C) $(n=2)$ alone or with 200 or $300 \mu \mathrm{M}$ of PCs inhibitor $(n=3)$. The complete system was incubated for a total of 4 days.

\section{Quantification of spheroid size and invaded area}

After the spheroids were embedded, cell invasion of the spheroid was monitored by digital photography using a Leica DM IL LED Fluo inverted light microscope (Leica DFC450C camera) at room temperature, with the Leica Application Suite (LAS V4.4). Images were acquired every day (day $0=$ time of embedding in collagen; pictures were taken immediately after embedding) using a $4 \mathrm{x} / 0.10$ objective. Image processing and quantification of spheroids and invasion areas were performed using in-house software. This in-house software takes into account cell density and not the limits of cell migration in the collagen matrix, which is observed independently. The implemented algorithm uses local fluctuations of the image intensity for automated estimation of the invasion magnitude. It is robust enough to handle micrographs of different generation methods and various qualities without the concept of an invasive front of the spheroids [26, 42]. Data of areas are normalized for each day to the relative size of day zero and transformed into the percentage of invasion.

\section{Apoptosis, necrosis and proliferation assay}

C6 cells were seeded into 96-well plates at $30 \%$ confluence with conditioned media (CM) from untreated or Poly (I:C)treated NR8383. These CM were supplemented or not with $100 \mu \mathrm{M}$ of PCs inhibitor or the same volume of DMSO. The assay was conducted for $72 \mathrm{~h}$. C6 cells were also treated with $600 \mu \mathrm{M}$ of TMZ as a positive control for apoptosis and $30 \mu \mathrm{M}$ of Taxol as a positive control for the decrease of proliferation. The Real Time-Glo Annexin V apoptosis and Necrosis reagent (JA1011, Promega) was then added. The luminescence signal corresponding to the exposure of phosphatidylserine on the outer leaflet of cell membranes and was monitored after $72 \mathrm{~h}$ using a 96-well plate reader. As well as fluorescence signal corresponding to the release of DNA. Proliferation was measured with the incorporation of BrdU into newly synthesized DNA of actively proliferating cells according to the manufacturer's instructions (BrdU Cell Proliferation ELISA Kit ab126556, Abcam).

\section{Macrophages reactivation quantification by Immunofluorescence and proteomic analysis on C6/ NR8383 spheroids and macrophages}

Mixed C6/NR8383 spheroids were generated as described before. They were treated with $10 \mu \mathrm{g} / \mathrm{mL}$ of Poly (I:C) or with $100 \mu \mathrm{M}$ of PCs inhibitor. Spheroids were also treated 
with CM from non-treated or from Poly (I:C)-treated macrophages supplemented or not with PCs inhibitor for $72 \mathrm{~h}$. For proteomic analysis, same treatment was performed on NR8383 cells. Spheroids and cells were lysed before FASP and LC-MS/MS analysis as described above. For immunofluorescence, mixed spheroids were rinsed with PBS before fixation with $4 \%$ paraformaldehyde for $1 \mathrm{~h}$ and then washed 3 times in PBS. They were implanted in gelatin $175 \mathrm{mg} / \mathrm{mL}$ and frozen at $-20^{\circ} \mathrm{C}$ and $-80{ }^{\circ} \mathrm{C}$. Cryostat (Leica Microsystems, Nanterre, France) was used to cut the spheroids with a thickness of $8 \mu \mathrm{m}$ per tissue section. The sections where then thaw-mounted on Polylysine-coated slides. Tissue sections were incubated in blocking buffer (0.01\% Triton, $1 \%$ Normal Donkey Serum (NDS) and $1 \%$ BSA in PBS) for $1 \mathrm{~h}$ to avoid nonspecific background staining. Mixed spheroids were then incubated overnight at $4{ }^{\circ} \mathrm{C}$ with primary antibodies directed against rabbit antiCD206 (1:1000; from Abcam), goat anti-arginase 1 (1:500; from Novus) and mouse anti-CD68 (1:50; from bio-rad). After 2 washes for $1 \mathrm{~min}$ in PBS/Tween $200.1 \%$ and 3 washes for $5 \mathrm{~min}$ in PBS, the mixed spheroids were incubated for $1 \mathrm{~h}$ at room temperature with secondary donkey anti-mouse antibody conjugated to Alexa Fluor 555 or with secondary donkey anti-rabbit or donkey anti-goat IgG antibody conjugated to Alexa Fluor 488 diluted in blocking buffer (dilution 1:200; Invitrogen, Carlsbad, CA, USA). Mixed spheroids were rinsed with PBS/Tween $200.1 \%$ and PBS and counterstained with Hoechst (1:10,000; from euromedex) for $20 \mathrm{~min}$ at $4{ }^{\circ} \mathrm{C}$. Finally, after the last PBS washing, spheroids were mounted on a slide with Dako Fluorescent Mounting Medium (Agilent Dako, Santa Clara, CA, USA). Control experiments were performed following the same immunostaining protocol without the primary antibody incubation.

\section{Data and software availability}

Macrophages proteomics data including MaxQuant files and annotated MS/MS have been deposited to the ProteomeXchange Consortium via the PRIDE partner repository with the dataset identifier PXD020258 with a Username: reviewer68417@ebi.ac.uk and Password: mRlwauBi.

\section{Results}

\section{Poly $(\mathrm{I}: \mathrm{C})$ triggers changes in the phenotype and function of macrophages}

A proteomic study was conducted to decipher the molecular impact of different concentrations $(5,10$ and $15 \mu \mathrm{g} / \mathrm{mL})$ of Poly(I:C) on macrophages. Shotgun proteomic analysis of
NR8383 macrophages yielded 1632 protein identifications across all the samples. As a criterion of significance, we applied an ANOVA test with a significance threshold of $p<$ 0.05. A heatmap was created from which 61 proteins showed a significant difference in LFQ expression between Poly (I:C)-treated NR8383 macrophages and non-treated macrophages (Fig. 2a, Supplementary Data 1). Two branches separate the treated to non-treated Poly (I:C) NR8383 macrophages. Macrophages treated with a concentration of $5 \mu \mathrm{g} / \mathrm{mL}$ Poly (I:C) presents an intermediate profile between non-treated and $10-15 \mu \mathrm{g} / \mathrm{mL}$ Poly (I:C) treated macrophages. Above $10 \mu \mathrm{g} / \mathrm{mL}$ Poly(I:C), the proteome of macrophages is completely different (Fig. 2a). In fact, among the differentially regulated proteins, 18 were downregulated in macrophages treated with 10 and $15 \mu \mathrm{g} /$ $\mathrm{mL}$ of Poly (I:C), including 11 $\beta$-Hydroxysteroid Dehydrogenase (11 $\beta$-HSD1), and Transmembrane glycoprotein NMB (Gpnmb) involved in the attenuation of the T cell response (Supplementary Data 1). We also identified, in this cluster of downregulated proteins, Legumain (Lgmn), Lysosomal acid lipase (Lipa) and Platelet glycoprotein 4 (CD36) which are associated with M2 anti-inflammatory polarization of macrophages. Docking protein 3 (DOK3), a proximal negative regulator of TLR signaling, was also found to be downregulated in macrophages after stimulation with 10 and $15 \mu \mathrm{g} / \mathrm{mL}$ of Poly (I:C) (Supplementary Data 1). Systems biology analyses of these downregulated proteins (Fig. 2b, cluster 1) confirm that proteins involved in T Cell response inhibition as well as in breast cancer and neoplasia inhibition are downregulated. In contrast, 43 proteins were upregulated in macrophages after a treatment with 10 and $15 \mu \mathrm{g} / \mathrm{mL}$ of Poly (I:C), including Antigen peptide transporter 1 and 2 (Tap1, Tap2) involved in MHC1 self-antigen presentation, NDRG1 and Interferoninduced GTP-binding protein Mx1 (Mx1), involved in the innate immune response, as well as Interferon-induced guanylate-binding protein 2 (Gbp2) and 2-5-oligoadenylate synthase-like protein 1 (Oasl) (Supplementary Data 1). We also identified, in the upregulated protein cluster, Nicotinamide phosphoribosyltransferase (Nampt) required for $\mathrm{T}$ cell-mediated immune responses, Nitric oxide synthase (NOS2) and long-chain acyl-CoA synthetase 1 (ACSL1), markers for the M1 pro-inflammatory phenotype. Rasrelated protein Rab-8B (Rab8b) which plays a key role in autophagy processes and Embigin (Emb) involved in cellular adhesion and migration were also found to be upregulated in Poly (I:C)-treated macrophages (Supplementary Data 1), as well as branched-chain-amino-acid aminotransferase (Bcat1) which controls metabolic reprogramming in activated macrophages. Systems biology analyses established that proteins involved in macrophages, innate immunity and thus in immune response activation are more expressed upon Poly(I:C) (Fig. 2b, cluster 2). 


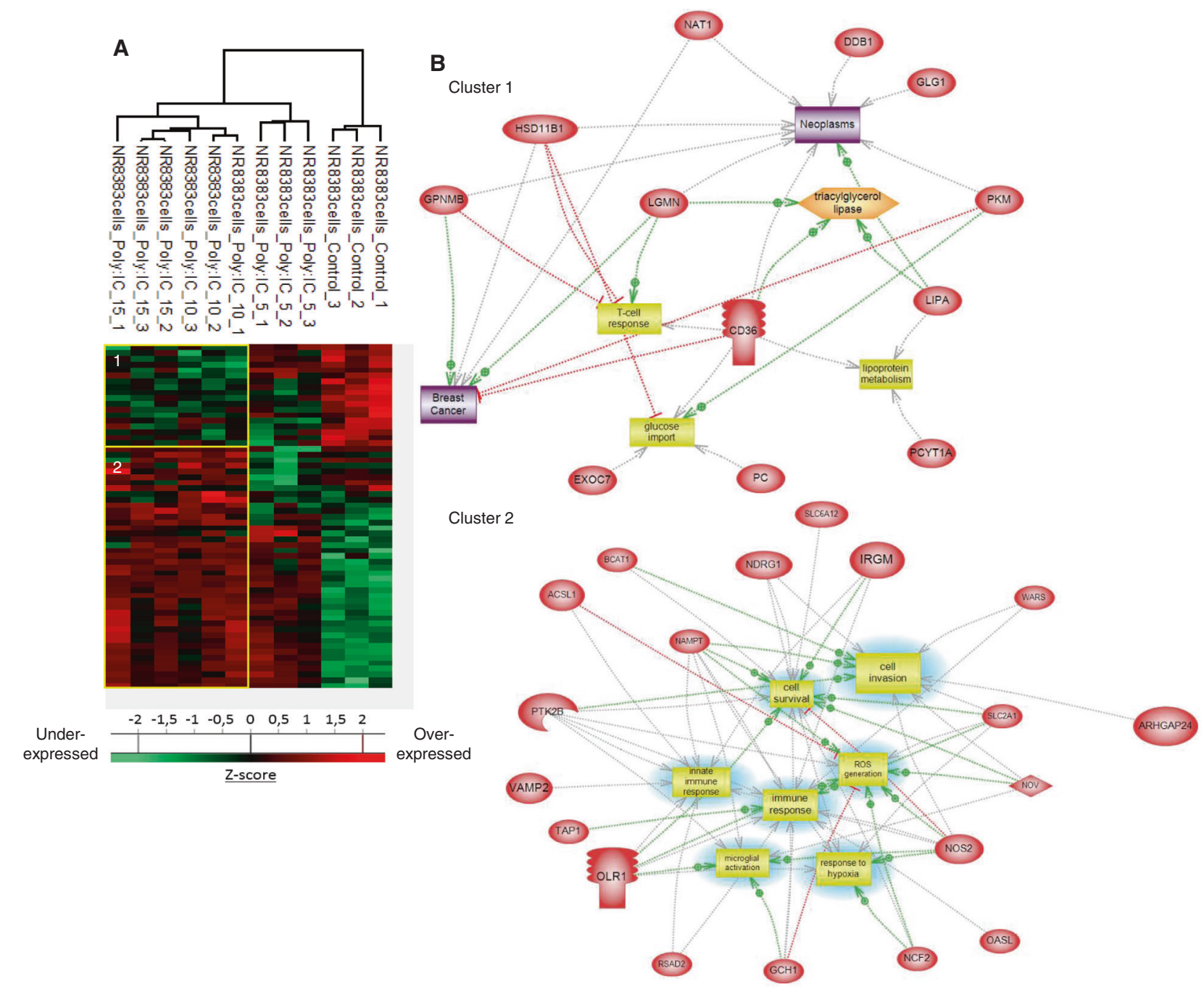

Fig. 2 Poly (I:C) induces an immune response by macrophages. a NR8383 macrophages were stimulated or not with Poly (I:C) $(5,10$ or $15 \mu \mathrm{g} / \mathrm{mL})(n=3)$. Cells were lysed before FASP and LC-MS/MS analysis. MaxQuant and Perseus software were used for the statistical analysis, and a heatmap was generated to show proteins that were significantly different between treated and untreated macrophages. Two clusters are highlighted. b Global pathway analyses of clusters 1 and 2.

\section{Poly $(\mathrm{I}: \mathrm{C})$ triggers the secretion of pro-inflammatory and anti-tumoral factors by macrophages}

The same approach was then applied to the conditioned medium of NR8383 treated with Poly (I:C) to analyse the secreted factors (Fig. 3). The proteomic analysis yielded a total of 845 proteins identified across all samples. 56 proteins showed a significant difference in LFQ expression between Poly (I:C)-treated NR8383 macrophages and nontreated macrophages (Supplementary Data 2). 16 proteins were downregulated after stimulation with all the concentration of Poly (I:C) compared to the untreated control (cluster 1, Fig. 3a). Among these, Sulfhydryl oxidase 1 (Qsox1), involved in tumor cell invasion and subsequent metastasis was found downregulated in cluster 1 such as CD166, TIMP2 and AGT knew to be involved in astrocytoma (Fig. 3b, cluster 1). The second cluster regroups 40 proteins which are more secreted by NR8383 macrophages after a stimulation with Poly (I:C), whatever the concentration used (cluster 2, Fig. 3a). Cytokines like CCL2, 3, 4 and 5 or CXCL10, are found in this cluster. These cytokines are important for immune cell recruitment, immune and inflammatory response. Several proteins involved in cell death-like Tumor necrosis factor (TNF), an anti-tumoral factor; Calpain-2 (CAPN2) and Cell division control protein 42 (CDC42) are also more secreted by NR8383 macrophages after a stimulation with Poly (I:C) (cluster 2, Fig. 3b). We also identified as upregulated, Fatty acid-binding protein (Fabp4), involved in the cellular response to the TNF and the inflammatory response, and also High mobility group protein B1 and B2 (Hmgb1 and Hmgb2) which play an important role during V(D)J 
A
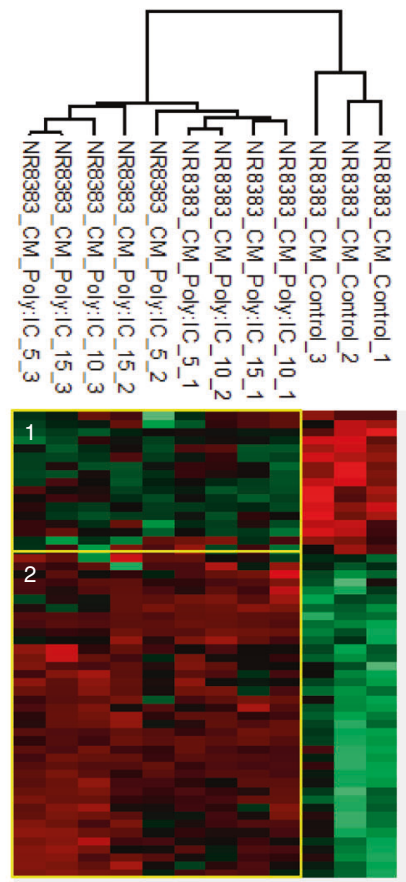

Underexpressed

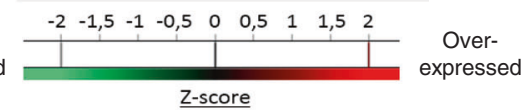

$\underline{\text { Z-score }}$

Fig. 3 Poly (I:C) induces secretion of pro-inflammatory cytokines by macrophages and immune-related proteins over-expression. a NR8383 macrophages were stimulated or not with Poly (I:C) $(5,10$ or $15 \mu \mathrm{g} / \mathrm{mL})(n=3)$. Conditioned media were digested and analyzed with LC-MS/MS. MaxQuant and Perseus software were used for the

recombination. Systems biology analyses (Fig. 3b, cluster 2) confirm that all up-regulated proteins found in this cluster can inhibit neoplasia and metastasis. Moreover, Kegg analyses point out that Poly (I:C) triggers proteomic changes both in the cellular component and in the secreted proteins of macrophages. We can observe a secretion of proinflammatory and anti-tumoral factors increased in Poly(I: C)-treated macrophages. We thus investigated the antitumoral ability of Poly (I:C) combined with PCs inhibitor which has previously demonstrated abilities to inactivate the tumor and activate macrophages [22].

Thereby, we performed an invasion assay on spheroids made of rat $\mathrm{C} 6$ glioma cell line and on mixed spheroids made of both C6 glioma and NR8383 rat macrophages (Fig. 4). The spheroids were cultured for 4 days with Poly (I:C) or with conditioned medium (CM) from Poly (I:C)-treated macrophages combined or not with PCs inhibitor. Their growth and invasion of the matrix by cells migrating out the initial core were monitored over these 4 days. Spheroids containing only C6 cells (Fig. 4a.i) were compared to spheroids containing a mix of C6 and NR8383 cells (Fig. 4a.ii). As we described before, we observed a decrease of spheroid growth and invasion after 2 days of treatment statistical analysis, and a heat map was generated to show proteins that were significantly different between treated and untreated macrophages. Two clusters are highlighted. b Global pathway analyses of clusters 1 and 2.

with PCs inhibitor for C6 spheroids and after 3 days of treatment for mixed C6/NR8383 spheroids. No changes in spheroid growth and invasion were observed after a treatment with Poly (I:C) alone compared to the control DMSO. The combination of Poly (I:C) and the PCs inhibitor decreases the invasion of spheroids, but the observed effect is not different from the effect of the inhibitor alone. However, we identified by mass spectrometry some antitumoral factors in the conditioned medium of Poly (I:C)treated macrophages (Fig. 3). Thus, to investigate the antitumoral activity of these factors we treated both types of spheroids with conditioned medium from macrophages previously treated or not with Poly (I:C). These conditioned media were associated or not with PCs inhibitor to assess the complementarity of both agents. C6 spheroids and mixed C6/NR8383 spheroids growth and invasion were decreased after 2 days of treatment with conditioned medium from Poly (I:C)-treated macrophages (Fig. 4b.i, 4b.ii). This effect increased progressively across time. Compared to the conditioned medium from untreated-macrophages, a statistical difference of more than $30 \%$ was observed after 4 days of treatment. No additive effect was observed when PCs inhibitor was combined with conditioned medium from 

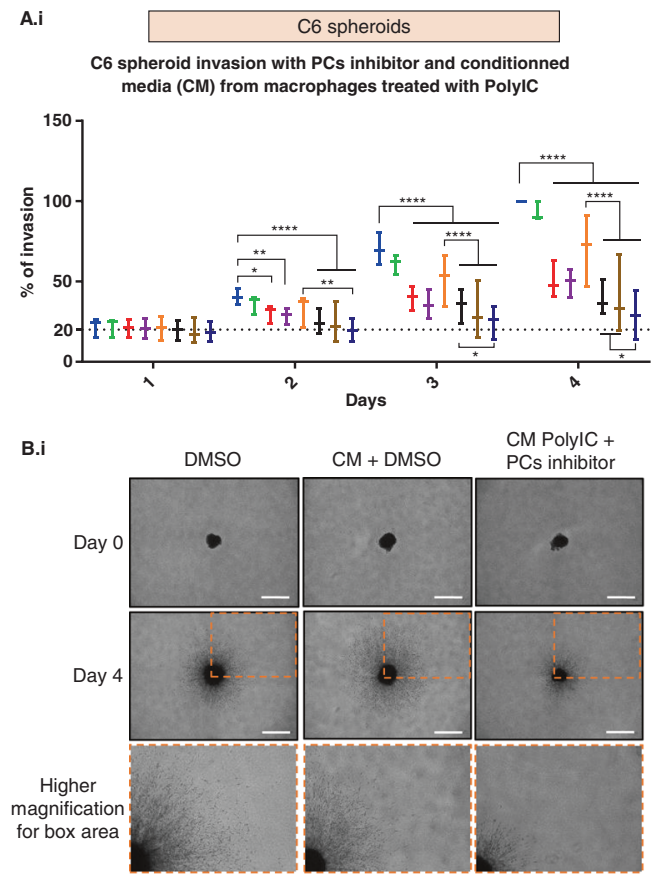

Fig. 4 PCs inhibitor associated with the conditioned media of Poly (I:C) activated macrophages has an anti-tumoral effect. C6 (i) and mixed C6/NR8383 (ii) spheroids were incubated with vehicle (DMSO), or with $10 \mu \mathrm{g} / \mathrm{ml}$ of Poly (I:C), or with $100 \mu \mathrm{M}$ of PCs inhibitor or with both Poly (I:C) and PCs inhibitor. Spheroids were also incubated with conditioned media (CM) from untreated macrophages complemented with PCs inhibitor or vehicle (DMSO) or from macrophages treated with Poly (I:C) for 24 h (i.e., CM Poly (I:C)) complemented with PCs inhibitor or vehicle (DMSO) $(n=3)$. Images of spheroids in the collagen matrix were taken every $24 \mathrm{~h}$ for 4 days. a Graphic representation showing the percentage of C6 (i) and mixed C6/NR8383 (ii) spheroids invasion. Significant differences were

Poly (I:C)-treated macrophages because of the low invasion rate of spheroids. Taken together, invasion assays and proteomic demonstrated secretion of anti-tumoral factors by macrophages after Poly (I:C) treatment.

Taken together, these results showed that Poly(I:C) treatment alone is not sufficient to trigger the anti-tumor activity of macrophages co-cultured with glioma cells whereas the conditioned medium of Poly(I:C) treated macrophages can inhibit glioma cells invasion. Thus, we next investigated the effect of macrophages Poly(I:C) pre-activation before their co-culture with glioma cells. As shown on Fig. 4c, pre-activated macrophages can keep their anti-tumoral activity and inhibit glioma cells invasion up to $50 \%$ (Fig. 4c).

We then investigated the impact of anti-tumoral factors secreted by macrophages on the $\mathrm{C} 6$ death and proliferation (Fig. 5). We measured the exposure of phosphatidylserine (PS) on the outer leaflet of cell membranes during the apoptotic process and used a DNA-binding dye to differentiate apoptosis from necrosis after $72 \mathrm{~h}$ of treatment (Fig. 5a). C6 glioma cells were treated with temozolomide (TMZ) as a positive control for apoptosis. Compared to the non-
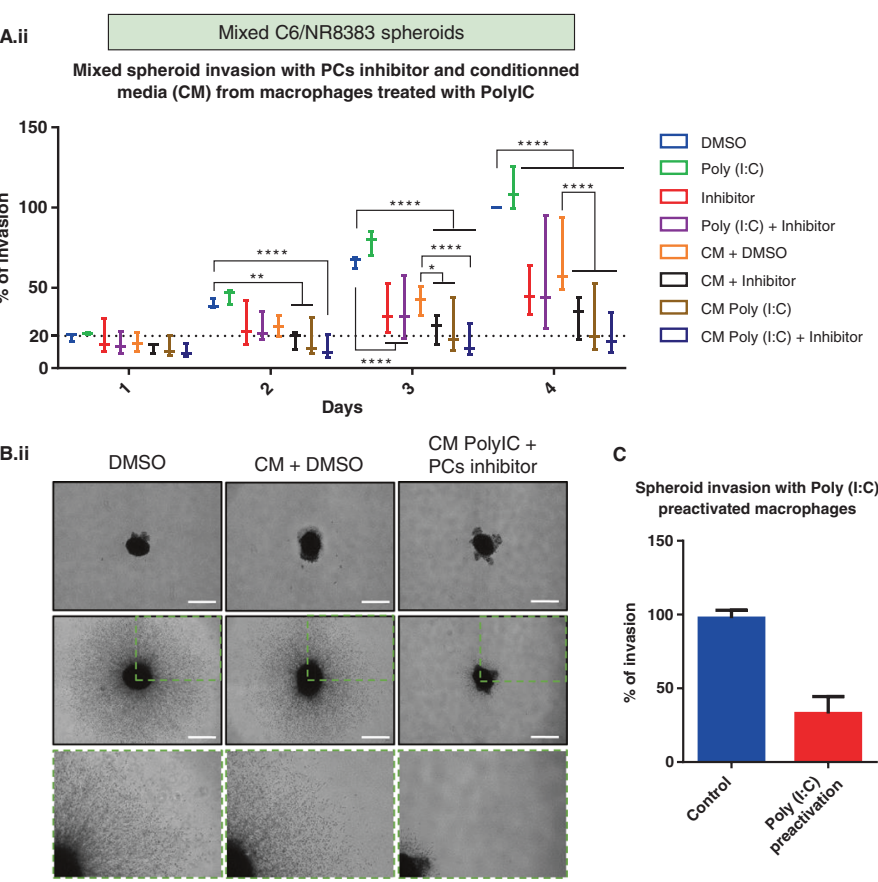

c

Spheroid invasion with Poly (l:C) preactivated macrophages

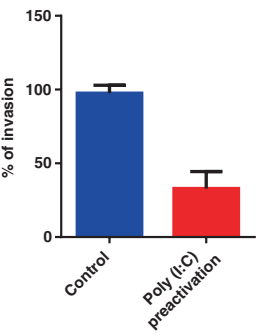

identified using Tukey's multiple comparisons test with $* * * * p<$ 0.0001 ; $* * p<0.001$; $* * p<0.01$. b Representative images of the invasion of C6 (i) spheroids and mixed C6/NR8383 (ii) spheroids in the collagen matrix at day 0 and day 4 . Spheroids were incubated with (DMSO), or with CM from untreated macrophages complemented with DMSO, or with CM from macrophages treated with Poly (I:C) for $24 \mathrm{~h}$ complemented with PCs inhibitor. All images were acquired with an inverted light microscope at $5 \mathrm{x}$ magnification. Scale bar: $500 \mu \mathrm{m}$. c NR8383 was pre-treated or not with $10 \mu \mathrm{g} / \mathrm{mL}$ of Poly (I:C) for $24 \mathrm{~h}$ and then used to create mixed spheroids with C6 glioma cells. Graphic representation showing the percentage of invasion at day $3(n=2)$.

treated cells and positive control, no signal for exposure of PS in C6 cells was recorded after the different treatments. However, a very high fluorescence signal due to the DNA dye was observed after a treatment with the conditioned medium from Poly (I:C) treated-macrophages, associated or not with the PCs inhibitor. This signal reflects a loss of membrane integrity and therefore cell death by necrosis. Moreover, a BrdU test correlates these results (Fig. 5b). Indeed, the proliferation of cells is decreased when the cells are treated with the conditioned medium from macrophages treated with Poly (I:C). This effect was more significant when the PCs inhibitor was associated with the conditioned medium from macrophages treated with Poly (I:C).

Finally, to know the approximative molecular weight of the anti-tumoral factors, we performed a viability assay of C6 cancer cells in presence of the different molecular weight fractions of the conditioned medium coming from Poly (I:C) stimulated or unstimulated macrophages. We identified that the fractions containing the anti-tumoral activity are the ones with a molecular weight ranging between $50-100 \mathrm{kDa}$ (Supplementary Fig. 1). 
A

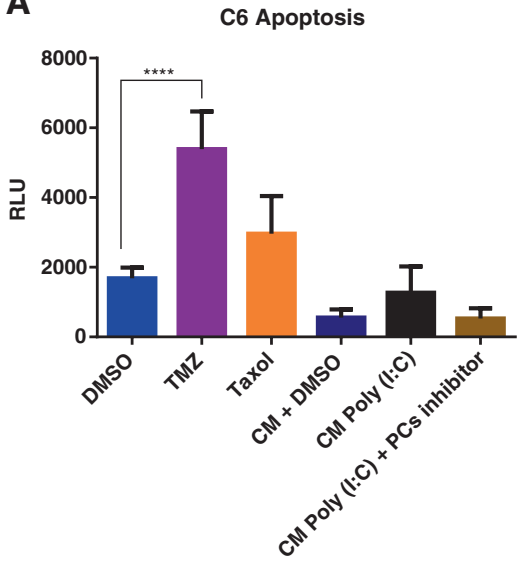

Fig. 5 Conditioned media from Poly (I:C) treated-macrophages leads to the $\mathrm{C6}$ glioma cells necrosis. $\mathrm{C} 6$ cell line was incubated with Poly (I:C) $(10 \mu \mathrm{g} / \mathrm{ml})$, PCs inhibitor $(100$ or $300 \mu \mathrm{M})$, TMZ $(600 \mu \mathrm{M})$, the conditioned media (CM) from untreated macrophages complemented with DMSO, the CM from Poly (I:C) pretreated macrophages complemented with DMSO, PCs Inhibitor $(100 \mu \mathrm{M})$ or with

\section{Poly (I:C) and PCs inhibitor are complementary for macrophages reactivation}

A proteomic study was conducted to decipher the molecular impact of the association between Poly (I:C) and PCs inhibitor on macrophages and their secretion (Figs. 6 and 7). First, shotgun proteomic analysis of NR8383 cells yielded 1,717 protein identification across all samples. As a criterion of significance, we applied an ANOVA test with a significance threshold of $p<0.05$. A heatmap was created from which 38 proteins showed a significant difference in LFQ expression between treated and non-treated NR8383 macrophages (Fig. 6a, Supp Data 3). Two branches separate the Poly (I:C) +/- PCs inhibitor-treated macrophages to untreated and PCs inhibitor-treated macrophages. Among these proteins, 7 were downregulated in macrophages treated with Poly (I:C), associated or not with PCs inhibitor (Fig. 6a, cluster 1). In this protein cluster, we found the transmembrane glycoprotein NMB (Gpnmb), and the Microphthalmia-associated transcription factor (Mitf), a transcription factor that regulates the expression of genes with essential roles in cell differentiation, proliferation and survival. 10 proteins are downregulated only after a treatment with Poly (I:C) associated with PCs inhibitor (Fig. 6a, cluster 2). Among these proteins, we found 2,5-phosphodiesterase 12 (Pde12) a negative regulator for antiviral and antitumor functions induced by interferons and Eukaryotic translation initiation factor 3 subunit E (Eif3e) which is essential for proliferation and survival of glioblastoma cells. In a third cluster, we found upregulated proteins after a treatment with Poly (I:C), associated or not with PCs inhibitor. In this cluster, we find several proteins previously described as upregulated after a Poly (I:C) treatment

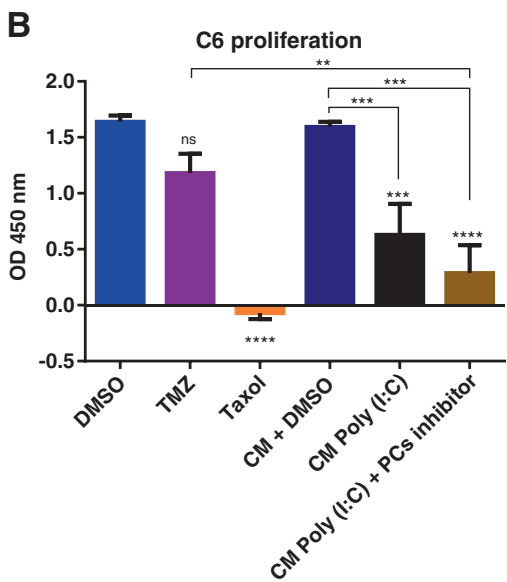

Taxol $(30 \mu \mathrm{M})$. a Necrosis was monitored with RealTime-Glo ${ }^{\mathrm{TM}}$ Apoptosis and Necrosis Assay. b The C6 proliferation was studied with BrDU incorporation. All the results were representative of three independent experiments. Significant differences were identified using Tukey's multiple comparisons test with $* * * * p<0.0001 ; * * * p<0.001$; $* * p<0.01 * p<0.05 ; n s=$ non significate.

(Fig. 2), as Gbp2, Tap1, Mx1 and Oasl involved in innate immune response and Nos2 marker for the M1 proinflammatory phenotype. We also found UDP-glucose 6dehydrogenase (Ugdh), a component of the extracellular matrix and F-actin-capping protein subunit alpha-1 (Capza1) which regulate actin cytoskeleton remodeling.

The same approach was then applied to the conditioned medium of NR8383 treated with Poly (I:C) associated or not with PCs inhibitor to analyse the secreted factors (Fig. 7, Supplementary Data 4). The proteomic analysis yielded a total of 1152 proteins identified across all samples. 66 proteins showed a significant difference in LFQ expression between treated NR8383 macrophages and nontreated macrophages. Two branches separate the PCs inhibitor $+/$ - Poly (I:C) treated macrophages to untreated and Poly (I:C) treated macrophages. Compared to the untreated control, 39 proteins were downregulated after stimulation with PCs inhibitor, associated or not with Poly (I:C). Conditioned medium from cells treated with only Poly (I:C) show the same expression profile than the untreated cells (Fig. 7a). The down-secretion by macrophages of migration-related protein macrophage migration inhibitory factor (MIF), also known to be involved in glioblastoma progression, was previously described after treatment with PCs inhibitor [22]. In this cluster, we also found Qsox1, as described in Fig. 3a. Several proteins involved in different tumorigenesis processes are down-secreted by macrophages after treatment with PCs inhibitor. Among these proteins, we identified Disintegrin and metalloproteinase domaincontaining protein 17 (Adam17), known to promotes glioma cell malignant phenotype and Branched-chainamino-acid aminotransferase (Bcat1) which promotes cell proliferation in glioma. Calcium/calmodulin-dependent 
A

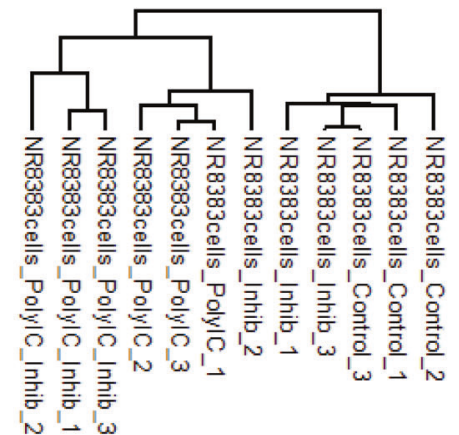

B Cluster 1

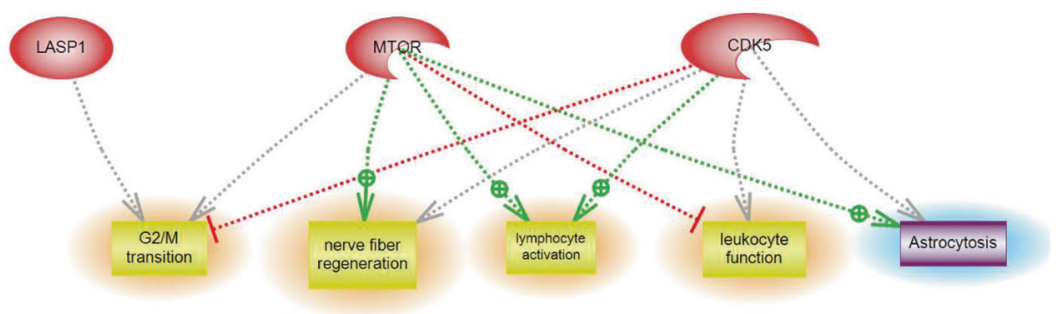

Cluster 3
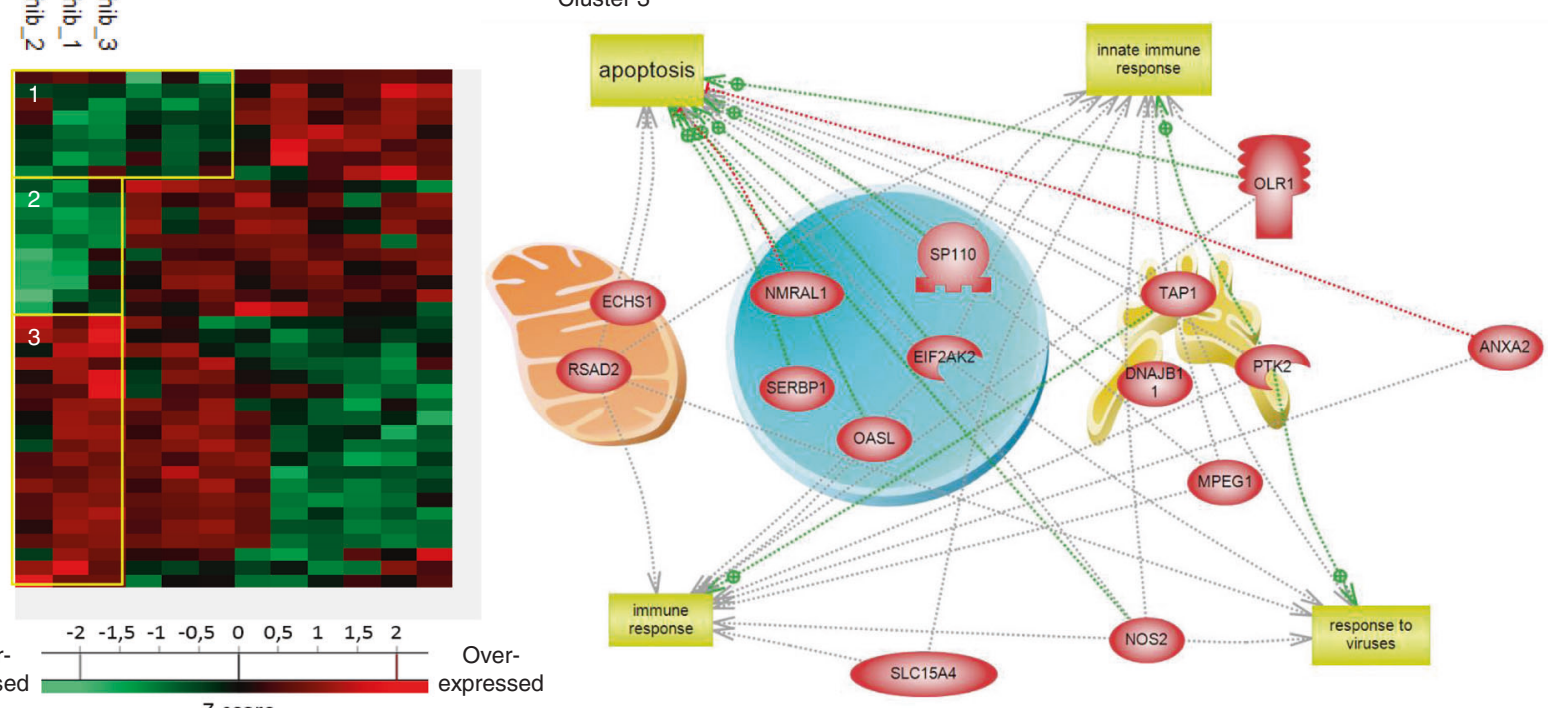

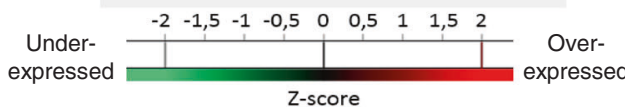

Fig. 6 Poly (I:C) associated with PCs inhibitor induces immunerelated proteins over-expression and underexpression of protumoral proteins. a NR8383 macrophages were stimulated with $10 \mu \mathrm{g} / \mathrm{mL}$ of Poly (I:C) associated or not with $100 \mu \mathrm{M}$ of PCs inhibitor $(n=3)$. Cells were lysed before FASP and LC-MS/MS analysis.

protein kinase type II subunit delta (Camk2d) plays a critical role in the invasion and metastasis properties of glioma cells and is down-secreted by macrophages after treatment with PCs inhibitor. Insulin-like growth factor II (Igf2) which plays a role in reprogramming macrophage into the anti-inflammatory phenotype was also down-secreted by macrophages after PCs inhibitor treatment. A second protein cluster can be highlighted in which we found 25 proteins over-expressed after a treatment with PCs inhibitor associated with Poly (I:C) (cluster 2, Fig. 7a). 12 of these are also over-expressed after treatment with PCs inhibitor but not after a treatment with Poly (I:C) (cluster 2.1, Fig. 7a). Among these proteins, we found Ubiquitin carboxylterminal hydrolase 15 (Usp15) which positively regulates type I interferon responses and thereby antiviral immune response. The other 13 proteins of the second cluster are over-expressed after a treatment with Poly (I:C) and after treatment with both Poly (I:C) and PCs inhibitor (cluster 2.2, Fig. 7a). Among these proteins, we found Cxcl10, Ccl3, Ccl4 and Tnf several cytokines described above (Fig. 3a). We also found, as described above (Fig. 3a) Gbp2
MaxQuant and Perseus software were used for the statistical analysis, and a heat map was generated to show proteins that were significantly different between treated and untreated macrophages. Three clusters are highlighted (1, 2 and 3). b Global pathway analyses of clusters 1 and 3 .

and Hnrnpa2b1, which initiate and amplify the innate immune response, and Inhba M1 pro-inflammatory phenotype marker.

Taken together, these results show a complementarity between Poly (I:C) and PCs inhibitor in the macrophages reactivation. The Poly (I:C) triggers more proteins changes in the cell component than the PCs inhibitor and, on the contrary, PCs inhibitor triggers more changes in the secretion profile of macrophages. Besides, several proteins show expression changes only when macrophages are stimulated with both Poly (I:C) and PCs inhibitor (cluster 2, Fig. 6a and cluster 2, Fig. 7a). Moreover, we showed with immunofluorescence on mixed macrophages-cancer cells spheroids a decrease in the expression of Arginase 1 and CD206, both markers of the pro-tumoral phenotype, when they were cultivated in presence of the conditioned medium from Poly (I:C)-treated macrophages. The numbers of CD206+ and arginase + macrophages seem to be lower when PCs inhibitor was combined with conditioned medium from Poly (I: C) treated macrophages (Fig. 8). To confirm these results, we analyzed the expression of pro-inflammatory and anti- 


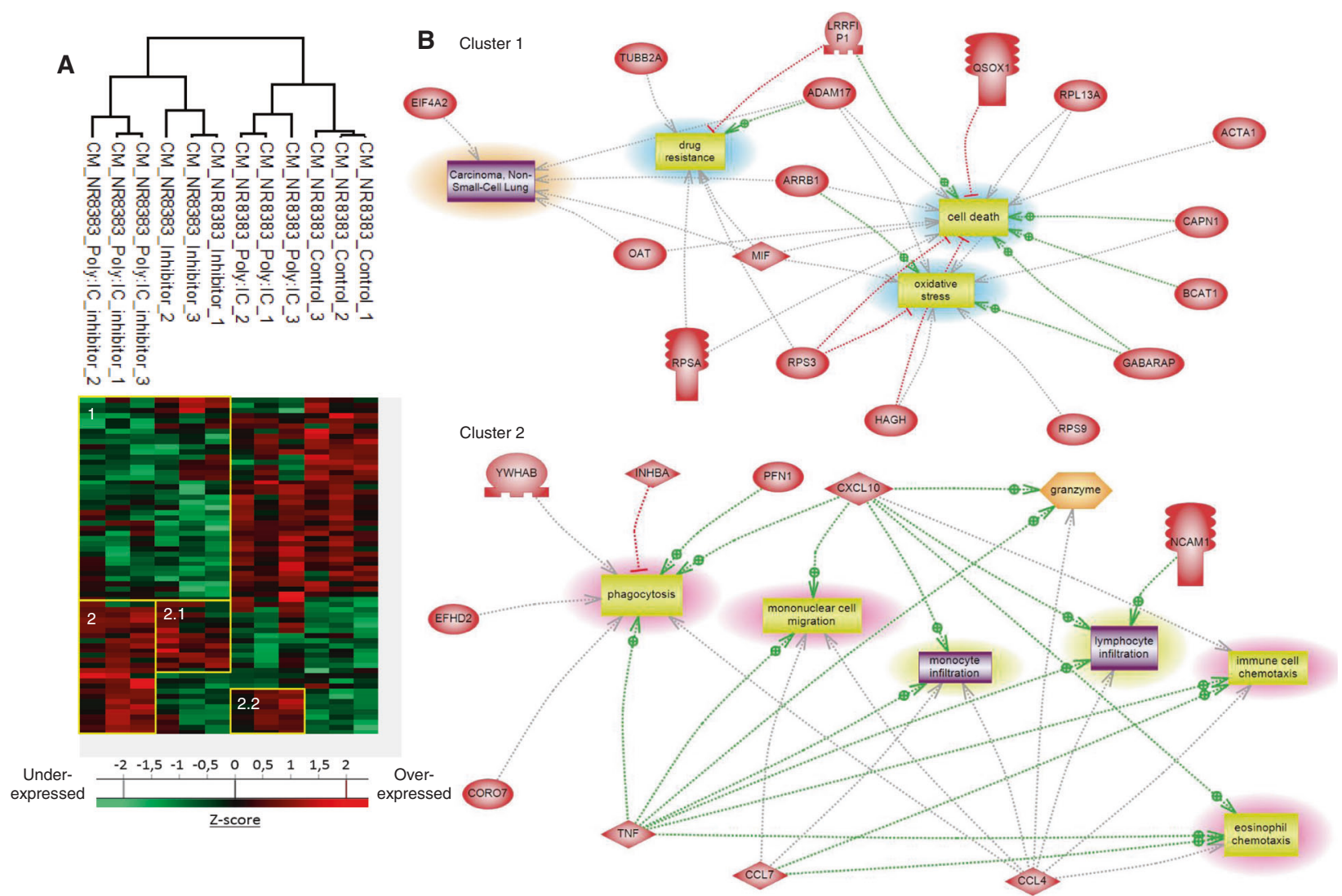

Fig. 7 Poly (I:C) associated with PCs inhibitor induces secretion of pro-inflammatory cytokines by macrophages. NR8383 macrophages were stimulated with $10 \mu \mathrm{g} / \mathrm{mL}$ of Poly (I:C) associated or not with $100 \mu \mathrm{M}$ of PCs inhibitor $(n=3)$. Conditioned media were digested and analyzed with LC-MS/MS. a MaxQuant and Perseus

inflammatory markers in the proteomic data (Supplementary Fig. 2). A decrease of anti-inflammatory markers expression is measured for CD206, STAT3 and Arginase 1 in macrophages treated with the conditioned medium from Poly (I:C)-treated macrophages with and without the addition of the PCs inhibitor. We also observed a higher expression of the pro-inflammatory marker MHC I in these macrophages even when they are associated with glioma cells in spheroids. We finally confirmed on spheroids made of NCH82 human glioma cells that the Poly (I:C) and PCs inhibitor are highly efficient on tumor invasion decrease 4 days after treatment (Supplementary Fig. 3).

\section{Discussion}

In the present study, we have demonstrated, based on shotgun proteomic studies, that Poly (I:C) at a concentration ranging between 10 and $15 \mu \mathrm{g} / \mathrm{mL}$ of Poly (I:C) changes the phenotype of macrophages. Their immune functions seem to be activated and their phenotype is close to a proinflammatory phenotype. A lower concentration $(5 \mu \mathrm{g} / \mathrm{mL})$ software were used for the statistical analysis, and a heatmap was generated to show proteins that were significantly different between treated and untreated macrophages. Two clusters are highlighted (1 and 2). b Global pathway analyses of clusters 1 and 2 .

is not enough to observe this activation state. This proinflammatory phenotype is based on an increase of expression of proteins involved in self-antigen presentation (Tap1, Tap2), the implication in anti-viral response through interferon intracellular signaling activation, Nitric oxide synthase (NOS2) and long-chain acyl-CoA synthetase 1 (ACSL1) level increase, as well as the involvement of factors such like Embigin known being implicated in cellular adhesion and migration or the branched-chain-aminoacid aminotransferase involved in metabolic reprogramming in activated macrophages. Similarly, analyses of the secreted factors revealed the presence of pro-inflammatory cytokines (CCL2, CCL3, CCL4 and CCL5 or CXCL10) and TNF. These results are in line with the ones obtained on rodent or human poly(I:C)-stimulated macrophage showing the production of TNF and or CXCL-10 (IP-10) [43, 44]. CXCL10 is known to play a significant role in leukocyte homing to inflamed tissues, increased production of CXCL10 may exacerbate inflammation [43]. Thus, augmented CXCL10 production by macrophages, may further exacerbate the inflammatory response and increase $\mathrm{T}$ cell recruitment which is expected in oncoimmune therapy. 
Fig. 8 Conditioned media from Poly (I:C) treated macrophages combine with PCs inhibitor decrease the Arginase expression into mixed spheroids. Mixed C6/ NR8383 spheroids were incubated for $24 \mathrm{~h}$ with vehicle (DMSO), or with $10 \mu \mathrm{g} / \mathrm{mL}$ of Poly (I:C) or with $100 \mu \mathrm{M}$ of PCs inhibitor $(n=3)$. Spheroids were also incubated with conditioned media (CM) from untreated macrophages complemented with vehicle (DMSO) or from macrophages treated with Poly (I:C) for $24 \mathrm{~h}$ (i.e., CM Poly (I:C)) complemented with PCs inhibitor. a Confocal images of mixed spheroids after cryosection and immunofluorescence staining for Hoechst (blue), CD68 (red) and Arginase 1 (green) are shown. b Percentage of macrophages (CD68) expressing arginase 1 or CD206. Significant differences were identified using $\mathrm{T}$ test with $* * p<0.01$ and $* p<0.05$.
A
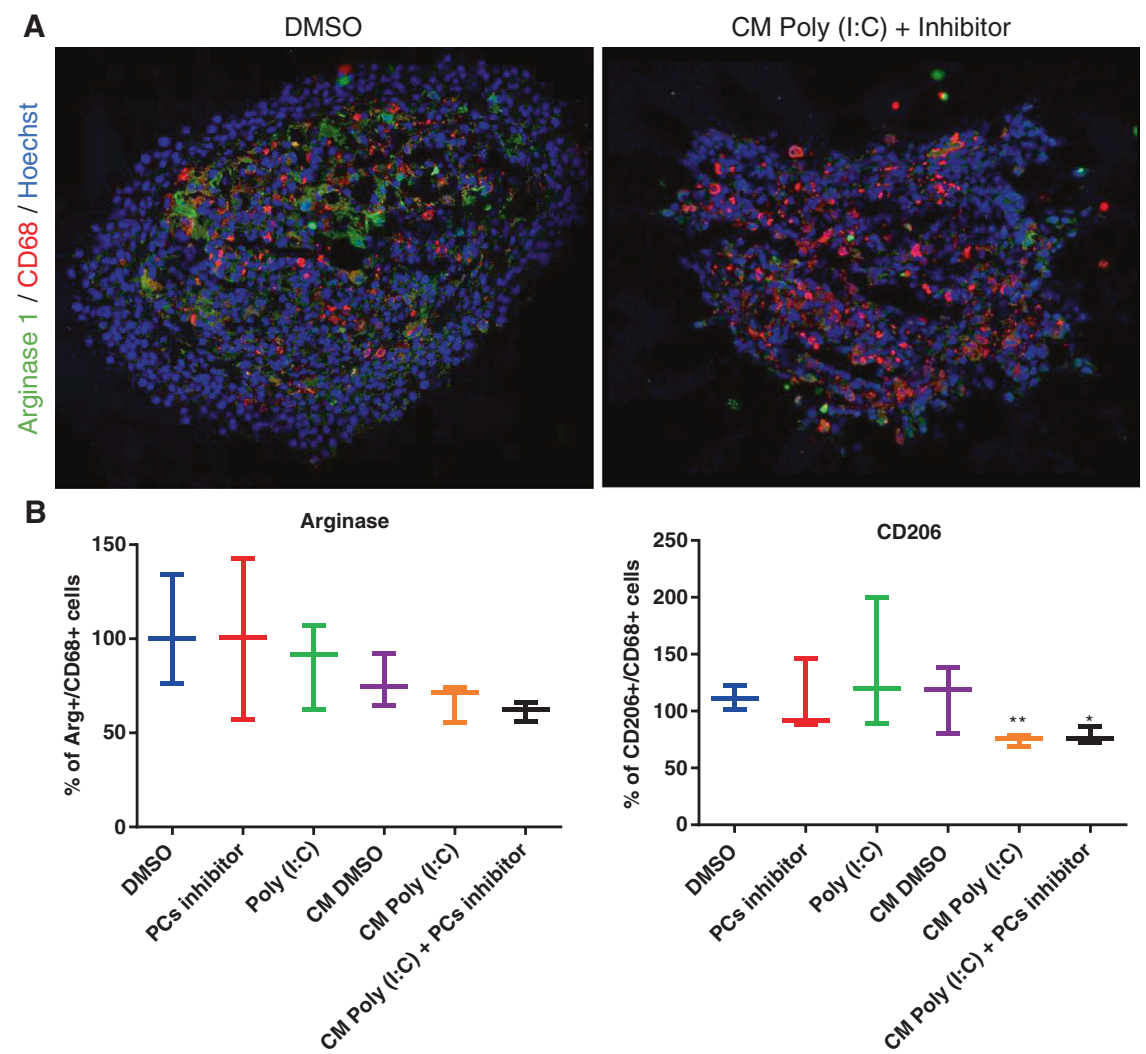

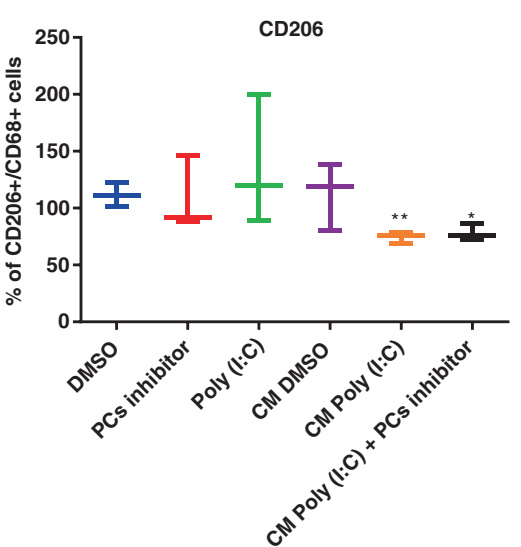

Based on these results, we tested the effects of such activated medium issued from Poly(I:C) treated macrophages on glioma cells in 3D culture. Compared to the conditioned medium from untreated-macrophages, a statistical difference of invasion of glioma cells of more than $30 \%$ was observed after 4 days of treatment. Thus, invasion assays and proteomic demonstrated secretion of anti-tumoral factors by macrophages after Poly (I:C) treatment. We confirmed the relevance of the conditioned medium from Poly (I:C) treated-macrophages with several biological essays. We established that glioma proliferation is decreased when the glioma cells are treated with the conditioned medium from macrophages treated with Poly (I:C). These results are in the same line as the ones of Maeda and collaborators [45]. This team has demonstrated that macrophages differentiated in the presence of pancreatic tumor cells (PANC1 and PT45) and treated with Poly (I:C) secrete more CXCL10 and CCL5 which trigger the cytotoxic activity of TC-M $\phi$ against cancer cells [45]. We have also demonstrated the efficacy of Poly (I:C) and PCs inhibitor on the invasion decrease of spheroids made with human glioma cell line NCH82. In our work, we identified that the fractions containing the anti-tumoral activity in the conditioned medium correspond to fractions with a molecular weight ranging between $50-100 \mathrm{kDa}$. We finally established using immunofluorescence studies with anti-CD68, anti-arginase and anti-CD206 that the combined treatment leads to the activation of TAMs with an M1 phenotype directly into the tumor spheroids whereas TAMs express an M2-like phenotype without treatment.

Altogether, we established that the combination of Poly (I:C) macrophages conditioned medium and the PCs inhibitor allowed the reactivation of tumor-associated macrophages which consequently inhibits tumor proliferation and invasion. The next step we will be to hunt in Poly (I:C) macrophages conditioned medium the anti-tumoral factors. These factors are secreted and could be contained in extracellular vesicles (EVs) as we previously showed with microglia cells $[16,46]$. In this context, an innovative therapeutic strategy based on EVs from Poly (I:C)-stimulated macrophages incorporating PCs inhibitor will be envisaged and needs further experiments.

Acknowledgements This research was supported by the funding from Ministère de l'enseignement Supérieur, de la Recherche et de l'innovation (MESRI), Institut National de la Santé et de la Recherche Médicale (Inserm), région Hauts-de-France and Université de Lille.

Author contributions Conceptualization, F.R. and M.S. Methodology, M.D., M.R., L.Z., A.R.V., M.S.; Validation, M.D., M.R. Formal analysis, M.S., M.R., M.D. Resources, M.S. I.F.; Data curation, M.D., M.S., S.A., F.R., M.R. Writing, M.S., M.D., M.R. Original draft, M.S., M.R., M.D. Writing - Review and Editing, M.S. M.D., F.R., I.F.; Supervision. M.S. Project Administration, M.S. 


\section{Compliance with ethical standards}

Conflict of interest The authors declare that they have no conflict of interest.

Publisher's note Springer Nature remains neutral with regard to jurisdictional claims in published maps and institutional affiliations.

Open Access This article is licensed under a Creative Commons Attribution 4.0 International License, which permits use, sharing, adaptation, distribution and reproduction in any medium or format, as long as you give appropriate credit to the original author(s) and the source, provide a link to the Creative Commons license, and indicate if changes were made. The images or other third party material in this article are included in the article's Creative Commons license, unless indicated otherwise in a credit line to the material. If material is not included in the article's Creative Commons license and your intended use is not permitted by statutory regulation or exceeds the permitted use, you will need to obtain permission directly from the copyright holder. To view a copy of this license, visit http://creativecommons. org/licenses/by/4.0/.

\section{References}

1. Thorsson V, Gibbs DL, Brown SD, Wolf D, Bortone DS, Ou Yang $\mathrm{T}-\mathrm{H}$, et al. The immune landscape of cancer. Immunity 2018;48:812-30.e14.

2. Heiland DH, Haaker G, Delev D, Mercas B, Masalha W, Heynckes S, et al. Comprehensive analysis of PD-L1 expression in glioblastoma multiforme. Oncotarget 2017;8:42214-25.

3. Mantovani A, Marchesi F, Malesci A, Laghi L, Allavena P. Tumour-associated macrophages as treatment targets in oncology. Nat Rev Clin Oncol 2017;14:399-416.

4. Jaaks P, Bernasconi M. The proprotein convertase furin in tumour progression. Int J Cancer 2017;141:654-63.

5. Seidah NG. The proprotein convertases in health and disease. Qatar Found Annu Res Forum Proc. 2012 Oct; (2012):AESNP11.

6. Day R, Salzet M The neuroendocrine phenotype, cellular plasticity, and the search for genetic switches: redefining the diffuse neuroendocrine system. Neuro Endocrinol Lett 2002. 23:447-51.

7. Duckert P, Brunak S, Blom N. Prediction of proprotein convertase cleavage sites. Protein Eng Des Sel 2004;17:107-12.

8. Scamuffa N, Calvo F, Chrétien M, Seidah NG, Khatib A-M. Proprotein convertases: lessons from knockouts. FASEB J 2006;20:1954-63.

9. Martín MG, Lindberg I, Solorzano-Vargas RS, Wang J, Avitzur Y, Bandsma R, et al. Congenital proprotein convertase 1/3 deficiency causes malabsorptive diarrhea and other endocrinopathies in a pediatric cohort. Gastroenterology 2013;145:138-48.

10. Marcinkiewicz M. $\beta A P P$ and furin mRNA concentrates in immature senile plaques in the brain of Alzheimer patients. J Neuropathol Exp Neurol 2002;61:815-29.

11. Khatib A-M, Siegfried G, Chrétien M, Metrakos P, Seidah NG. Proprotein convertases in tumor progression and malignancy. Am J Pathol 2002 Jun;160:1921-35.

12. Vergara D, Merlot B, Lucot JP, Collinet P, Vinatier D, Fournier I, et al. Epithelial-mesenchymal transition in ovarian cancer. Cancer Lett 2010;291:59-66.

13. Simeone P, Trerotola M, Franck J, Cardon T, Marchisio M, Fournier I, et al. The multiverse nature of epithelial to mesenchymal transition. Vol. 58, Seminars in Cancer Biology. Academic Press; 2019. p. 1-10.

14. Longuespée R, Couture F, Levesque C, Kwiatkowska A, Desjardins R, Gagnon S, et al. Implications of proprotein convertases in ovarian cancer cell proliferation and tumor progression: insights for PACE4 as a therapeutic target. Transl Oncol 2014;7:410.

15. D'Anjou F, Routhier S, Perreault J-P, Latil A, Bonnel D, Fournier I, et al. Molecular validation of PACE4 as a target in prostate cancer. Transl Oncol 2011;4:157-72.

16. Murgoci A-N, Cizkova D, Majerova P, Petrovova E, Medvecky L, Fournier I, et al. Brain-cortex microglia-derived exosomes: nanoparticles for glioma therapy. Chemphyschem. 2018;19:1205-14.

17. Coppola JM, Bhojani MS, Ross BD, Rehemtulla A. A smallmolecule furin inhibitor inhibits cancer cell motility and invasiveness. Neoplasia 2008;10:363-70.

18. Levesque C, Couture F, Kwiatkowska A, Desjardins R, Guérin B, Neugebauer WA, et al. PACE4 inhibitors and their peptidomimetic analogs block prostate cancer tumor progression through quiescence induction, increased apoptosis and impaired neovascularisation. Oncotarget. 2015;6:3680-93.

19. Ghisoli M, Barve M, Mennel R, Lenarsky C, Horvath S, Wallraven $\mathrm{G}$, et al. Three-year follow up of GMCSF/bi-shRNA furin DNAtransfected autologous tumor immunotherapy (Vigil) in metastatic advanced Ewing's sarcoma. Mol Ther 2016;24:1478-83.

20. Oh J, Barve M, Matthews CM, Koon EC, Heffernan TP, Fine B, et al. Phase II study of Vigil ${ }^{\circledR}$ DNA engineered immunotherapy as maintenance in advanced stage ovarian cancer. Gynecol Oncol 2016;143:504-10.

21. Lapierre M, Siegfried G, Scamuffa N, Bontemps Y, Calvo F, Seidah NG, et al. Opposing function of the proprotein convertases furin and PACE4 on breast cancer cells' malignant phenotypes: Role of tissue inhibitors of metalloproteinase-1. Cancer Res. 2007;67:9030-4.

22. Rose M, Duhamel M, Aboulouard S, Kobeissy F, Le Rhun E, Desmons A, et al. The role of a proprotein convertase inhibitor in reactivation of tumor-associated macrophages and inhibition of glioma growth. Mol Ther 2020;17:31-46.

23. Lansac G, Dong W, Dubois CM, Benlarbi N, Afonso C, Fournier $\mathrm{I}$, et al. Lipopolysaccharide mediated regulation of neuroendocrine associated proprotein convertases and neuropeptide precursor processing in the rat spleen. J Neuroimmunol 2006;171:57-71.

24. Refaie S, Gagnon S, Gagnon H, Desjardins R, D'Anjou F, D'Orléans-Juste $\mathrm{P}$, et al. Disruption of proprotein convertase $1 / 3$ $(\mathrm{PC} 1 / 3)$ expression in mice causes innate immune defects and uncontrolled cytokine secretion. J Biol Chem 2012;287:14703-17.

25. Gagnon H, Refaie S, Gagnon S, Desjardins R, Salzet M, Day R Proprotein convertase $1 / 3(\mathrm{PC} 1 / 3)$ in the rat alveolar macrophage cell line NR8383: localization, trafficking and effects on cytokine secretion. PLoS ONE. 2013;8(4).

26. Duhamel M, Rose M, Rodet F, Murgoci AN, Zografidou L, Régnier-Vigouroux $\mathrm{A}$, et al. Paclitaxel treatment and proprotein convertase $1 / 3(\mathrm{PC} 1 / 3)$ knockdown in macrophages is a promising antiglioma strategy as revealed by proteomics and cytotoxicity studies. Mol Cell Proteom. 2018;17:1126-43.

27. Duhamel M, Rodet F, Delhem N, Vanden Abeele F, Kobeissy F, Nataf S, et al. Molecular consequences of proprotein convertase 1/ $3(\mathrm{PC} 1 / 3)$ inhibition in macrophages for application to cancer immunotherapy: a proteomic study. Mol Cell Proteom 2015;14:2857-77.

28. Duhamel M, Rodet F, Murgoci AN, Desjardins R, Gagnon H, Wisztorski $\mathrm{M}$, et al. The proprotein convertase $\mathrm{PC} 1 / 3$ regulates TLR9 trafficking and the associated signaling pathways. Sci Rep. 2016;6:1-13.

29. Rodet F, Capuz A, Ozcan B-A, Le Beillan R, Raffo-Romero A, Kobeissy F, et al. PC1/3 KD Macrophages exhibit resistance to the Inhibitory effect of IL-10 and a higher TLR4 activation rate, leading to an anti-tumoral phenotype. Cells. 2019;8:1-19.

30. Pesu M, Watford WT, Wei L, Xu L, Fuss I, Strober W, et al. Tcell-expressed proprotein convertase furin is essential for maintenance of peripheral immune tolerance. Nature 2008;455:246-50. 
31. Oksanen A, Aittomäki S, Jankovic D, Ortutay Z, Pulkkinen K, Hämäläinen S, et al. Proprotein convertase FURIN constrains Th2 differentiation and is critical for host resistance against Toxoplasma gondii. J Immunol 2014;193:5470-9.

32. Kees T, Lohr J, Noack J, Mora R, Gdynia G, Tödt G, et al. Microglia isolated from patients with glioma gain antitumor activities on poly (I:C) stimulation. Neuro Oncol 2012;14: 64-78.

33. Wiśniewski JR, Zougman A, Nagaraj N, Mann M. Universal sample preparation method for proteome analysis. Nat Methods 2009;6:359-62.

34. Wiśniewski JR Filter-Aided Sample Preparation. In: Methods in Enzymology. 2017. p. 15-27.

35. Wiśniewski JR. Quantitative evaluation of filter aided sample preparation (FASP) and multienzyme digestion FASP protocols. Anal Chem 2016;88:5438-43.

36. Cox J, Mann M. MaxQuant enables high peptide identification rates, individualized p.p.b.-range mass accuracies and proteomewide protein quantification. Nat Biotechnol 2008;26:1367-72.

37. Tyanova S, Temu T, Cox J. The MaxQuant computational platform for mass spectrometry-based shotgun proteomics. Nat Protoc 2016;11:2301-19.

38. Cox J, Neuhauser N, Michalski A, Scheltema RA, Olsen JV, Mann M. Andromeda: a peptide search engine integrated into the MaxQuant environment. J Proteome Res 2011;10:1794-805.

39. Cox J, Hein MY, Luber CA, Paron I, Nagaraj N, Mann M. Accurate proteome-wide label-free quantification by delayed normalization and maximal peptide ratio extraction, termed MaxLFQ. Mol Cell Proteom 2014;13:2513-26.

40. Tyanova S, Temu T, Sinitcyn P, Carlson A, Hein MY, Geiger T, et al. The Perseus computational platform for comprehensive analysis of (prote)omics data. Nat Methods 2016;13:731-40.

41. Yuryev A, Kotelnikova E, Daraselia N. Ariadne's chemEffect and pathway studio knowledge base. Expert Opin Drug Discov 2009;4:1307-18.

42. Cisneros Castillo LR, Oancea AD, Stüllein C, Régnier-Vigouroux A, et al. A novel computer-assisted approach to evaluate multicellular tumor spheroid invasion assay. Sci Rep. 2016;6:1-16.

43. Lyons C, Fernandes P, Fanning LJ, Houston A, Brint E. Engagement of Fas on macrophages modulates poly I:C induced cytokine production with specific enhancement of IP-10. Spencer J, editor. PLoS ONE 2015;10:e0123635.

44. Cui WY, Zhao S, Polanowska-Grabowska R, Wang J, Wei J, Dash B, et al. Identification and characterization of Poly(I:C)induced molecular responses attenuated by nicotine in mouse macrophages. Mol Pharmacol 2013;83:61-72.

45. Maeda A, Digifico E, Andon FT, Mantovani A, Allavena P. Poly (I:C) stimulation is superior than Imiquimod to induce the antitumoral functional profile of tumor-conditioned macrophages. Eur J Immunol 2019;49:801-11.

46. Murgoci AN, Duhamel M, Raffo-Romero A, Mallah K, Aboulouard S, Lefebvre C, et al. Location of neonatal microglia drives small extracellular vesicles content and biological functions in vitro. J Extracell Vesicles. 2020;9:1-18. 